\title{
Qualitative evaluation of EBSD technique for the study of plastic deformation
}

Jennifer Cocle $^{*}$, Pierre Hovington ${ }^{* *}$, Marin Lagacé ${ }^{* *}$ and Raynald Gauvin ${ }^{*}$.

* Department of Mining, Metals and Materials Engineering, McGill University, Montréal, Québec, Canada, H3A 2B2.

Institut de Recherche d'Hydro-Québec (IREQ), Varennes, Québec, Canada, J3X 1S1.

Electron back-scattered diffraction (EBSD) system is growing in popularity because of the practical and economic aspects that it shows : possibility of several analyses in one map (macro- and micro-texture analysis, grain boundaries analysis, grains size analysis, etc.), bulk material analysis, better statistical results, easier handling, etc. Therefore, the application of EBSD technique to the study of plastic deformation mechanisms could facilitate and accelerate the microstructural analysis associated with the research and development of materials. Preliminary tests were thus carried out with the INCA system of the Oxford Company, installed in a Hitachi S-4700 Fe-SEM, in order to evaluate the potential of EBSD technique for the study of plastic deformation mechanisms. The sample analyzed in this study is a rolled plate of electrolytic copper.

Two examples of EBSD results are presented on figure 1: the image quality (fig.1b) and misorientation angles (fig.1c and 1d) associated with the specific sample area showed in figure 1a. The image quality is related to the intensity gradients of EBSP whereas the misorientation angles result from the indexing process performed by the INCA software (comparison of experimental EBSPs with those initially contained in the software). An interesting fact of these results touches the potential coincidence of the zones of low intensity of the image quality (black points, fig. 1b) with the zones of low misorientation angles (green points, interval 2.5 to $15^{\circ}$, fig. 1c and 1d). Indeed, points of low intensity are typical of deformed crystallographic structures. In parallel, a grain is, by definition, constituted of a uniform (same) crystallographic structure. Consequently, the presence of misorientation inside the grains (green points) might be associated with an increase of dislocations density inside the grains. However, it is important to mention that certain factors can generate on EBSPs effects similar to those of plastic deformation : widening and loss of clearness of the Kikuchi bands, loss of contrast of EBSP, etc. [1-6]. These factors are related to the microscope conditions (acceleration voltage, beam current, surface contamination, etc.), the EBSD software (integration time, indexing error, video gain, etc.), the sample preparation (residual stresses and roughness of the sample surface, scratches, corrosion pits, etc.) and the material itself (atomic number, second phases, work hardening, etc.). Figure 2 shows an example of these effects for the acceleration voltage of the microscope. It is noticed that a reduction of the acceleration voltage seems to generate some blurring of the EBSPs. In this way, these factors must be recognised in the analysis of the results of figure 1 . The use of statistical methods and images techniques analysis might allow the identification and quantification of the influent factors on image quality and indexing process in order to validate the characterization of dislocations using EBSD. 


\section{REFERENCES}

[1] F. J. Humphreys, Journal of Materials Science 36 (2001) 3833-3854.

[2] A. D. Rollet et al., Crystallographic information via microtexture techniques, in: Advanced Characterization and Microstructural Analysis, 2003.

[3] A. J. Schwartz et al., Electron Backscatter Diffraction in Materials Science, Klumar Academic/Plenum Publishers, New York, 2000, p. 339.

[4] R. A. Schwarzer, Micron 28 (1997) 249-264.

[5] A. J. Wilkinson, D. J. Dingley, Acta Metallurgica et Materialia 39 (1991) 3047-3055.

[6] A. J. Wilkinson, P. B. Hirsch, Micron 28 (1997) 279-308.

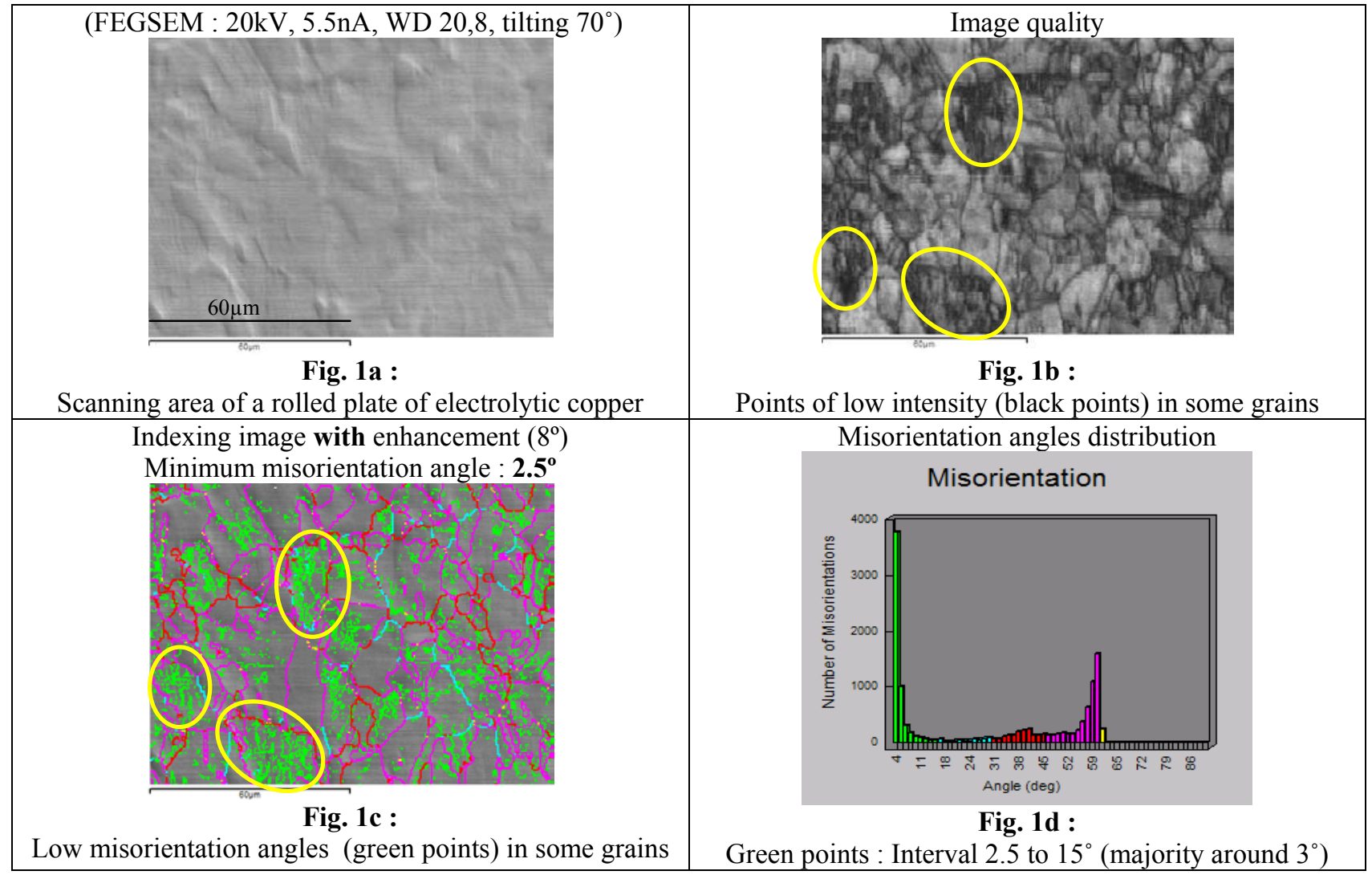

FIG. 1 : EBSD results for the study of plastic deformation mechanisms of electrolytic copper.

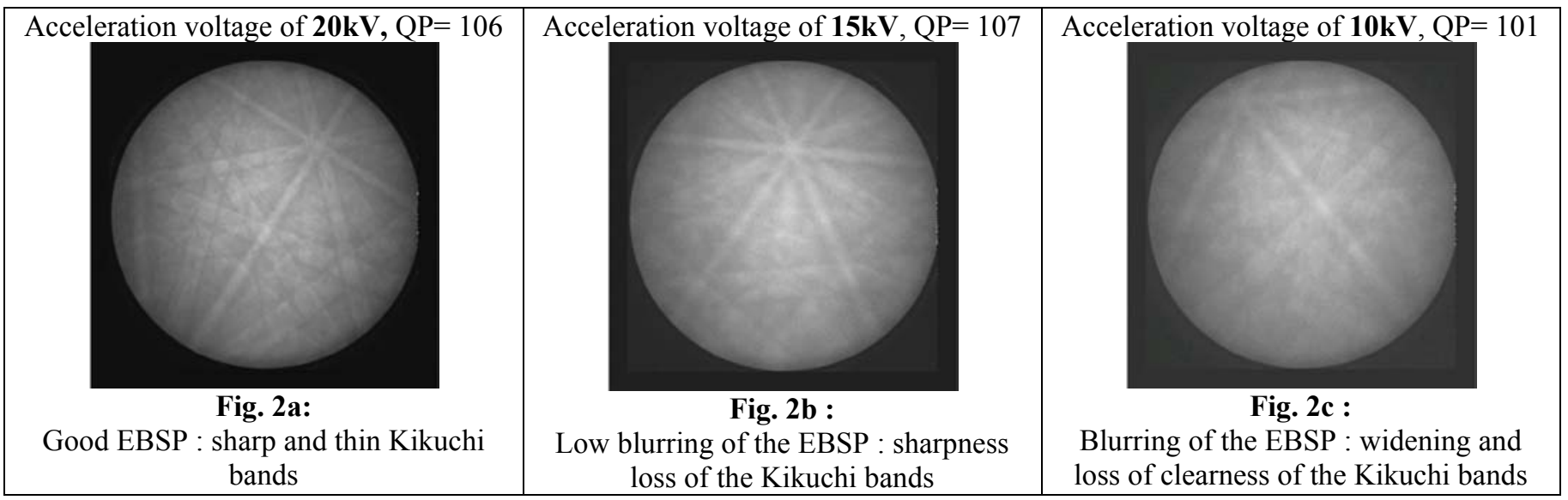

FIG. 2 : Acceleration voltage effect on electrolytic copper EBSPs. 